\title{
Predição do potencial genético de populações segregantes de arroz de terras altas ${ }^{(1)}$
}

\author{
Patrícia Guimarães Santos ${ }^{(2)}$, Antônio Alves Soares ${ }^{(3)}$ e Magno Antonio Patto Ramalho(4)
}

\begin{abstract}
Resumo - O objetivo deste trabalho foi avaliar o método de Jinks \& Pooni na predição do potencial genético de populações segregantes de arroz e estudar o efeito da interação populações segregantes $\mathrm{x}$ ambiente na seleção destas populações. Utilizaram-se 23 populações segregantes de arroz de terras altas e duas testemunhas, avaliadas em um látice 5x5, com três repetições, no ano agrícola de 1996/97. O estudo foi conduzido em dois locais em Minas Gerais, Lavras e Patos de Minas, em três épocas distintas de semeadura. Os resultados encontrados em relação à probabilidade de extrair linhagens superiores a um determinado padrão indicaram como mais promissoras as populações CNAx $5496 \mathrm{e}$ CNAx 6001, e menos promissoras, CNAx 6063 e CNAx 6102. A previsão do potencial genético das populações segregantes a partir do método de Jinks \& Pooni mostrou-se uma alternativa viável na escolha das populações mais promissoras, permitindo ao melhorista concentrar maiores esforços na avaliação das famílias superiores. A ocorrência das interações populações segregantes x épocas e locais x épocas mostraram a importância de se avaliar as populações em mais de um ambiente. A escolha das populações que apresentam um comportamento estável frente às oscilações ambientais é importante dentro de um programa de melhoramento.
\end{abstract}

Termos para indexação: Oryza sativa, interação genótipo-ambiente, época de semeadura, métodos de melhoramento.

\section{Prediction of genetic potential of segregating upland rice population}

\begin{abstract}
This work aimed to evaluate the Jinks \& Pooni's method to predict genetic potential of the segregating upland rice population and to study the effect of the segregating population $\mathrm{x}$ environment interaction in the selection of those populations. In this work 23 segregating populations of upland rice with two checks were used. They were evaluated in a $5 \times 5$ lattice, with three replicates, in the agricultural year of 1996/97. The populations were conducted in two sites in Minas Gerais State, Brazil, Lavras and Patos de Minas, at three distinct sowing times. The results found in relation to the probability of extracting superior lines from a certain population indicated as the most promising the populations CNAx 5496 and CNAx 6001 and as the least promising CNAx 6063 and CNAx 6102. The Jinks $\&$ Pooni's method was a feasible alternative in the choice of populations most promising permitting the breeder to concentrate his effort in the evaluation of a higher family. The occurrence of the segregating population $\mathrm{x}$ sowing time and site $\mathrm{x}$ sowing time interactions showed the importance of evaluating the populations in more than one environment. The choice of the populations which presented a steady behavior against the environmental drifts is an important step within an improvement program and the chance to obtain success in this phase rises.
\end{abstract}

Index terms: Oryza sativa, genotype-environment interaction, sowing date, breeding methods.

\footnotetext{
(1) Aceito para publicação em 20 de julho de 2000 .

(2) Universidade Federal de Uberlândia (UFU), Instituto de Ciências Agrárias, Caixa Postal 593, CEP 38400-902 Uberlândia, MG. E-mail: pgsantos@umuarama.ufu.br

(3) Universidade Federal de Lavras (UFLA), Dep. de Agricultura, Caixa Postal 37, CEP 37200-000 Lavras, MG E-mail: aasoares@ufla.br

${ }^{(4)}$ UFLA, Dep. de Biologia. E-mail: magnoapr@ufla.br
}

\section{Introdução}

Na condução de um programa de melhoramento por hibridação, o melhorista deve tomar algumas decisões. A primeira delas e de maior importância, da qual depende o sucesso de todo o trabalho de seleção, é a escolha das populações segregantes. É que além da sua performance média, a variabilidade exis- 
tente é fundamental, porque a população segregante obtida poderá expressar pequena variabilidade genética, que ocorre quando os genitores superiores que foram cruzados apresentarem constituições genéticas semelhantes para o caráter em apreço (Ramalho et al., 1993). A população segregante ideal é aquela que associa média alta e suficiente variabilidade genética que possibilite a seleção de linhagens com desempenho superior aos pais.

Entre vários procedimentos que podem ser utilizados na escolha das populações segregantes, um deles é o método de Jinks \& Pooni (1976). Este método permite estimar a probabilidade de se obter linhagens superiores a um determinado padrão na $\mathrm{F}_{\infty}$, utilizando para isso estimativas da média e variância de gerações iniciais. As informações que confirmam a sua eficiência já foram obtidas em espécies como fumo (Toledo, 1986), trigo (Snape, 1982), soja (Toledo, 1987, 1989; Triller, 1994) e feijão (Otubo, 1994; Abreu, 1997). Contudo, com arroz, não foi encontrado relato de sua utilização, o que mostra a necessidade de novas pesquisas para avaliar a eficiência do método nessa cultura.

Também importante em programa de seleção é o efeito do ambiente e sobretudo da interação genótipos $\mathrm{x}$ ambientes, que pode mascarar a expressão genotípica e não permitir progressos com a seleção. A época de semeadura do arroz no Brasil é muito ampla, estendendo-se de outubro a dezembro e às vezes até janeiro. Além disso, o arroz é cultivado em vários ambientes, que diferem em condições de fertilidade do solo, clima e principalmente no nível tecnológico empregado pelos agricultores. No cultivo de qualquer espécie em condições tão diversas de ambiente é esperada uma acentuada interação de genótipos x ambientes. A presença dessa interação tem sido constatada em experimentos de arroz principalmente com linhas puras (Morais et al., 1981, 1982). Contudo, são restritas as informações a respeito da interação em populações segregantes. Desta forma, é importante verificar se a ocorrência da interação populações segregantes $\mathrm{x}$ ambientes pode afetar o processo de identificação das populações a serem trabalhadas pelos melhoristas de arroz.

Este trabalho teve como objetivo avaliar o método de Jinks \& Pooni (1976) na escolha de populações segregantes de arroz de terras altas e verificar quais as implicações da interação populações x ambientes.

\section{Material e Métodos}

Foram utilizadas 23 populações segregantes de arroz de terras altas, desenvolvidas pela Embrapa-Centro Nacional de Pesquisa de Arroz e Feijão (CNPAF), a partir de cruzamentos biparentais e múltiplos (Tabela 1). Desde 1993, Minas Gerais tem recebido todos os anos populações $\mathrm{F}_{2}$ de vários cruzamentos, as quais foram avançadas pelo método de bulk, inicialmente em Lavras. As populações estudadas neste trabalho encontravam-se nas gerações $\mathrm{F}_{3}, \mathrm{~F}_{4}$ e $\mathrm{F}_{5}$ (Tabela 1)

Os experimentos foram instalados no ano agrícola de 1996/97, em dois locais, Lavras e Patos de Minas, nas Fazendas Experimentais da Empresa de Pesquisa Agropecuária de Minas Gerais (EPAMIG). Foram utilizadas três épocas de semeadura, na primeira quinzena dos meses de outubro, novembro e dezembro. O delineamento utilizado foi um látice $5 \times 5$, com três repetições, e duas testemunhas: Guarani e Canastra. As parcelas foram constituídas de duas linhas de cinco metros de comprimento e espaçadas de $40 \mathrm{~cm}$ na densidade de 50 sementes por metro.

A adubação de plantio constou de $400 \mathrm{~kg} / \mathrm{ha}$ da fórmula 4-30-16 + Zn e em torno de 45 dias após a semeadura procedeu-se à adubação de cobertura com uma aplicação de $150 \mathrm{~kg} / \mathrm{ha}$ de sulfato de amônio. Os experimentos foram submetidos à irrigação por aspersão após decorridos períodos prolongados de estiagem. Aos 30 dias após a emergência das plântulas, foi realizado desbaste para diminuir o número de plantas por metro linear e facilitar a colheita de plantas individuais. Os demais tratos culturais realizados foram os recomendados para a cultura do arroz de sequeiro.

Os caracteres avaliados foram: florescimento (dia), altura de plantas $(\mathrm{cm})$ e produtividade de grãos $(\mathrm{kg} / \mathrm{ha})$. Antes da colheita de toda a parcela foram amostradas aleatoriamente 15 plantas por parcela, em duas repetições, para avaliação da produtividade de grãos em g/planta para cada população.

A análise de variância foi realizada para cada caráter nas três épocas e cada local, considerando os efeitos de populações como sendo aleatórios. Posteriormente, foi efetuada a análise de variância conjunta envolvendo os dois locais e as três épocas. No modelo estatístico, consideraram-se os efeitos de populações e épocas como aleatórios e locais como fixos

A partir das esperanças dos quadrados médios foram estimados os componentes de variância e os parâmetros genéticos e fenotípicos entre as populações. Posteriormen- 
te, foram obtidas as estimativas de herdabilidade no sentido amplo, coeficiente de variação genético e o índice de variação b dessas populações, segundo Vencovsky \& Barriga (1992). Para estudar o efeito da interação entre populações segregantes x épocas de semeadura, foi utilizado procedimento alternativo proposto por Cruz \& Castoldi (1991) que permitiu decompor a interação duas a duas em parte simples e complexa.

A herdabilidade no sentido amplo da produtividade de grãos foi estimada a partir das variâncias genéticas $\left(\sigma^{2}{ }_{G}\right) \mathrm{e}$ fenotípicas $\left(\sigma_{\mathrm{F}}^{2}\right)$ das 15 plantas amostradas por parcela das populações segregantes e das testemunhas, de acordo com o método de Melo et al. (1997), que sugerem estimar a variância ambiental de cada população segregante a partir da média dos coeficientes de variação ambiental das testemunhas.

A previsão do potencial das populações foi feita a partir do procedimento de Jinks \& Pooni (1976), que estima a probabilidade de a população sob avaliação originar linhagens que superem um determinado padrão. Essa probabilidade corresponde à área direita ou esquerda de um determinado valor de $\mathrm{x}$ na abcissa da distribuição normal. Para determinar essa área foi utilizada a estimativa da ordenada $\mathrm{Z}$ pela expressão:

$\mathrm{Z}=\frac{\left(\overline{\mathrm{L}}-\overline{\mathrm{F}}_{\mathrm{n}}\right)}{\sqrt{\sigma_{\mathrm{FL}}^{2}}}$

em que,

$\overline{\mathrm{L}}$ : média considerada padrão; neste trabalho utilizou-se $\mathrm{L}=18,0 \mathrm{~g} /$ planta, valor superior à maior média encontrada entre as populações avaliadas;

$\overline{\mathrm{F}}_{\mathrm{n}}$ : média das linhagens na geração $\mathrm{F}_{\infty}$ que, em um modelo sem dominância, corresponde à média de qualquer geração segregante;

$\sqrt{\sigma_{\mathrm{FL}}^{2}}$ : variância fenotípica entre as linhagens

A variância genética entre as linhagens $\left(\sigma^{2}\right)$ corresponde a duas vezes a variância genética aditiva $\left(\sigma^{2} \mathrm{~A}\right)$ presente na $\mathrm{F}_{2}$. Em um modelo sem dominância, a variância fenotípica $\left(\sigma^{2} \mathrm{~F}\right)$ para as gerações avaliadas neste trabalho foram as seguintes:

Tabela 1. Relação das populações segregantes de arroz de sequeiro com seus respectivos genitores e geração.

\begin{tabular}{lll}
\hline População & Genitores & Geração \\
\hline CNAx 5482 & CNA 6186/IAC 84-198//Cuiabana/IRAT 336 & $\mathrm{F}_{5}$ \\
CNAx 5493 & Caiapó/A12-282-3//CNA 7691 & $\mathrm{F}_{5}$ \\
CNAx 5494 & Caiapó/Guarani G57A//Cuiabana/IRAT 336 & $\mathrm{F}_{5}$ \\
CNAx 5496 & Caiapó/IAC 84-198//CNA 7680 & $\mathrm{F}_{5}$ \\
CNAx 5500 & Caiapó/CNA 6673//CNA 7754 & $\mathrm{F}_{5}$ \\
CNAx 5883 & CNAx 3031-13-8-1-1/IAC 25R//CNA 7455/150055 & $\mathrm{F}_{4}$ \\
CNAx 5901 & IACx 25R/CNA 7013-0//15005/IAC 201 & $\mathrm{F}_{4}$ \\
CNAx 5939 & Chorinho MG/CNA 7455//IAC 25R/Lemont & $\mathrm{F}_{4}$ \\
CNAx 5945 & CNA 7455/Colombia 1//Rio Doce/IAC 25R & $\mathrm{F}_{4}$ \\
CNAx 5949 & CNA 7455/IRAT 112R//New Bonnet/Lemont & $\mathrm{F}_{4}$ \\
CNAx 5983 & IRAT 112R/L 90-28//Colombia 1/IAC 201 & $\mathrm{F}_{4}$ \\
CNAx 5987 & Carajá/IAC 25R//Lemont/IAC 201 & $\mathrm{F}_{4}$ \\
CNAx 6190 & L 141/CNA 8198 & $\mathrm{F}_{3}$ \\
CNAx 5995 & CNAx 5275-1/L 141 & $\mathrm{F}_{3}$ \\
CNAx 5996 & CNAx 5311-1/IAC 1364 & $\mathrm{F}_{3}$ \\
CNAx 6067 & CNA 8078/IAC 1364 & $\mathrm{F}_{3}$ \\
CNAx 6090 & Progresso/IAC 1203 & $\mathrm{F}_{3}$ \\
CNAx 6102 & BSL/CNA 8217 & $\mathrm{F}_{3}$ \\
CNAx 6106 & CNA 8200/IAC 1203 & $\mathrm{F}_{3}$ \\
CNAx 6001 & CNAx 4442-6-1-B-1/4001.BC 92-93 & $\mathrm{F}_{3}$ \\
CNAx 6062 & CNAx 8055/IAC 1203 & $\mathrm{F}_{3}$ \\
CNAx 6063 & CNAx 1722-9-1-1-1/L 141 & $\mathrm{F}_{3}$ \\
CNAx 6065 & CNA 8052/IAC 1365 & $\mathrm{F}_{3}$ \\
\hline
\end{tabular}




$$
\begin{aligned}
& \mathrm{F}_{3} \Rightarrow \sigma_{\mathrm{F}}^{2}=1,5 \sigma_{\mathrm{A}}^{2}+\sigma_{\mathrm{E}}^{2} \Rightarrow \sigma_{\mathrm{A}}^{2}=\frac{\sigma_{\mathrm{F}}^{2}-\sigma_{\mathrm{E}}^{2}}{1,5} \\
& \mathrm{~S}=\sqrt{\sigma_{\mathrm{FL}}^{2}}=\sqrt{2 \sigma_{\mathrm{A}}^{2}+\sigma_{\mathrm{E}}^{2}}=\sqrt{1,33 \sigma_{\mathrm{F}}^{2}-0,3 \sigma_{\mathrm{E}}^{2}} ; \\
& \mathrm{F}_{4} \Rightarrow \sigma_{\mathrm{F}}^{2}=1,75 \sigma_{\mathrm{A}}^{2}+\sigma_{\mathrm{E}}^{2} \Rightarrow \sigma_{\mathrm{A}}^{2}=\frac{\sigma_{\mathrm{F}}^{2}-\sigma_{\mathrm{E}}^{2}}{1,75} \\
& \mathrm{~S}=\sqrt{\sigma_{\mathrm{FL}}^{2}}=\sqrt{2 \sigma_{\mathrm{A}}^{2}+\sigma_{\mathrm{E}}^{2}}=\sqrt{1,14 \sigma_{\mathrm{F}}^{2}-0,14 \sigma_{\mathrm{E}}^{2}} ; \\
& \mathrm{F}_{5} \Rightarrow \sigma_{\mathrm{F}}^{2}=1,88 \sigma_{\mathrm{A}}^{2}+\sigma_{\mathrm{E}}^{2} \Rightarrow \sigma_{\mathrm{A}}^{2}=\frac{\sigma_{\mathrm{F}}^{2}-\sigma_{\mathrm{E}}^{2}}{1,88} \\
& \mathrm{~S}=\sqrt{\sigma_{\mathrm{FL}}^{2}}=\sqrt{2 \sigma_{\mathrm{A}}^{2}+\sigma_{\mathrm{E}}^{2}}=\sqrt{1,06 \sigma_{\mathrm{F}}^{2}-0,06 \sigma_{\mathrm{E}}^{2}} .
\end{aligned}
$$

O método utilizado para o estudo de adaptabilidade e estabilidade das populações foi o de Annicchiarico (1992). Esse método estima o índice de confiança $\left(\mathrm{I}_{\mathrm{i}}\right)$ de uma determinada cultivar apresentar desempenho abaixo da média do ambiente. Ele consiste em expressar as médias dos genótipos como porcentagem da média ambiental.

\section{Resultados e Discussão}

Com exceção de altura de planta em Lavras na segunda época, que diferiu significativamente a $5 \%$ de probabilidade, os caracteres avaliados apresentaram diferenças altamente significativas $(\mathrm{P}<0,01)$ nas três épocas de semeadura e nos dois locais estudados (Tabela 2). Isso indica a presença de variabilidade genética entre as populações avaliadas, que pode ser explorada pelo melhorista com o intuito de obter linhagens de arroz que se mostrem superiores às cultivares comumente utilizadas. Maiores coeficientes de variação ambiental $\left(\mathrm{CV}_{\mathrm{e}}\right)$ foram obtidos para produtividade de grãos, o que é esperado, já que esse caráter é o mais influenciado pelo ambiente. Esses resultados estão de acordo com os obtidos por Santos (1996).

A eficiência da utilização do delineamento em látice é mostrada também na Tabela 2. Observa-se que houve variação da sua eficiência de uma época para outra e de um local para outro. Por exemplo, na segunda época de semeadura, em Patos de Minas, o látice mostrou-se eficiente para todos os caracteres, ao passo que na terceira época, ele não seria necessário. Em Lavras, o látice foi eficiente em todas as épocas de semeadura para a maioria dos caracteres. Esses resultados mostram a importância de se adotar esse tipo de delineamento, principalmente quando o número de tratamentos é elevado e a área experimental é heterogênea.
Comparando-se as três épocas de semeadura, constatou-se que a produtividade de grãos foi superior em Lavras somente na primeira época, coincidindo com a maior altura de plantas. A semeadura nessa época foi favorecida nos dois locais, pois o florescimento da maioria dos genótipos coincidiu com a alta precipitação pluvial ocorrida no mês de janeiro. A severidade de brusone foi mais baixa, o que provavelmente esteve relacionado com a menor pressão de inóculo e com a maior disponibilidade de água no solo, tornando os materiais mais tolerantes.

$\mathrm{Na}$ segunda época de semeadura, as diferenças entre locais para os caracteres produtividade de grãos, altura de plantas e florescimento foram muito pequenas (Tabela 2). A floração das populações na segunda época de semeadura ocorreu em fevereiro, quando a precipitação foi baixa nos dois locais. Na terceira época, a superioridade das populações quanto à produtividade de grãos foi evidente em Patos de Minas, coincidindo novamente com a maior altura de plantas e o florescimento precoce dos genótipos, provavelmente em virtude de maior precipitação pluvial durante a floração. A seleção de linhagens precoces e produtivas tem sido um dos principais objetivos do programa de melhoramento genético do arroz de sequeiro do Estado de Minas Gerais, e os resultados deste trabalho revelam a viabilidade de se obter linhagens que atendam às exigências desse programa.

As estimativas da herdabilidade de todos os caracteres avaliados para as populações nas três épocas e nos dois locais oscilaram de médio a alto, indicando a possibilidade de o melhorista obter sucesso com a seleção (Tabela 2). Essa estimativa contém no numerador além da $\sigma_{\mathrm{A}}^{2}$ a estimativa de $\sigma^{2} \mathrm{D}$, o que pode torná-la superestimada, já que não foi possível isolar esses componentes. Contudo, para a cultura do arroz, existe a predominância da variância aditiva, sendo a variância de dominância menos expressiva, o que torna as estimativas da $\mathrm{h}^{2}$ mais confiáveis (Lopes, 1984; Ahmad et al., 1986; Morais, 1992).

$O$ índice de variação $b$, que é a relação entre o coeficiente de variação genético e o coeficiente de variação ambiental, segundo Vencovsky \& Barriga (1992) quando atinge valores superiores a 1,0 indica uma situação favorável à seleção. Dos caracteres avaliados, em Lavras, altura de plantas, nas segunda e terceira épocas, e produtividade de grãos, na se- 
Tabela 2. Resumo das análises de variância relativa à primeira, segunda e terceira época de semeadura para os caracteres produtividade de grãos (kg/ha), altura de plantas (cm) e florescimento (dia) em Lavras e Patos de Minas, MG. 1996/97.

\begin{tabular}{|c|c|c|c|c|c|c|c|}
\hline Caráter & Local & Fonte de variação & Quadrado médio & $\mathrm{CV}_{\mathrm{e}}$ & Média & $\mathrm{h}^{2}$ & $\mathrm{~b}$ \\
\hline & & & \multicolumn{5}{|c|}{ Primeira época da semeadura } \\
\hline \multirow{6}{*}{$\begin{array}{l}\text { Produtividade } \\
\text { de grão }\end{array}$} & Lavras & Trat. não aj. & $1.873 .353,7$ & 20,3 & 3.171 & 77,9 & 1,08 \\
\hline & & Erro $\mathrm{DBC}^{(1)}$ & $413.184,1$ & & & & \\
\hline & & Ef. látice $(\%)$ & - & & & & \\
\hline & P. de Minas & Trat. ajustado & $470.683,5^{* *}$ & 10,9 & 2.396 & 85,6 & 1,41 \\
\hline & & Erro efetivo & $67.645,3$ & & & & \\
\hline & & Ef. látice $(\%)$ & 109,4 & & & & \\
\hline \multirow{6}{*}{$\begin{array}{l}\text { Altura } \\
\text { de plantas }\end{array}$} & Lavras & Trat. ajustado & $290,9 * *$ & 7,5 & 110,7 & 76,3 & 1,03 \\
\hline & & Erro efetivo & 69,1 & & & & \\
\hline & & Ef. látice (\%) & 100,1 & & & & \\
\hline & P. de Minas & Trat. não aj. & $165,6^{* *}$ & 4,9 & 95,3 & 87,1 & 1,50 \\
\hline & & Erro DBC & 21,4 & & & & \\
\hline & & Ef. látice $(\%)$ & - & & & & \\
\hline \multirow[t]{6}{*}{ Florescimento } & Lavras & Trat. não aj. & $345,0 * *$ & 4,6 & 96,0 & 94,4 & 2,37 \\
\hline & & Erro DBC & 19,4 & & & & \\
\hline & & Ef. látice $(\%)$ & - & & & & \\
\hline & P. de Minas & Trat. ajustado & $207,9 * *$ & 2,0 & 89,6 & 98,5 & 8,04 \\
\hline & & Erro efetivo & 3,2 & & & & \\
\hline & & Ef. látice (\%) & 103,4 & & & & \\
\hline & & & \multicolumn{5}{|c|}{ Segunda época da semeadura } \\
\hline \multirow{6}{*}{$\begin{array}{l}\text { Produtividade } \\
\text { de grão }\end{array}$} & Lavras & Trat. ajustado & $1.259 .559,4^{* *}$ & 25,0 & 2.827 & 60,2 & 0,71 \\
\hline & & Erro efetivo & $501.290,6$ & & & & \\
\hline & & Ef. látice (\%) & 100,1 & & & & \\
\hline & P. de Minas & Trat. ajustado & $1.684 .596,6^{* *}$ & 12,1 & 2.944 & 92,5 & 2,03 \\
\hline & & Erro efetivo & $126.189,4$ & & & & \\
\hline & & Ef. látice (\%) & 381,2 & & & & \\
\hline \multirow{6}{*}{$\begin{array}{l}\text { Altura } \\
\text { de plantas }\end{array}$} & Lavras & Trat. ajustado & $133,2^{*}$ & 7,6 & 100,4 & 56,8 & 0,66 \\
\hline & & Erro efetivo & 57,5 & & & & \\
\hline & & Ef. látice (\%) & 114,2 & & & & \\
\hline & P. de Minas & Trat. ajustado & $201,04 * *$ & 6,1 & 101,2 & 81,3 & 1,20 \\
\hline & & Erro efetivo & 37,7 & & & & \\
\hline & & Ef. látice (\%) & 255,3 & & & & \\
\hline \multirow[t]{6}{*}{ Florescimento } & Lavras & Trat. ajustado & & 3,8 & 93,8 & 93,1 & 2,12 \\
\hline & & Erro efetivo & 12,5 & & & & \\
\hline & & Ef. látice (\%) & 100,1 & & & & \\
\hline & P. de Minas & Trat. ajustado & & 3,3 & 95,4 & 91,9 & 1,95 \\
\hline & & Erro efetivo & 9,8 & & & & \\
\hline & & Ef. látice (\%) & 150,5 & & & & \\
\hline \multirow{7}{*}{$\begin{array}{l}\text { Produtividade } \\
\text { de grão }\end{array}$} & & & \multicolumn{5}{|c|}{ Terceira época de semeadura } \\
\hline & Lavras & Trat. ajustado & $876.650,4^{* *}$ & 17,5 & 2.294 & 81,5 & 1,21 \\
\hline & & Erro efetivo & $161.930,6$ & & & & \\
\hline & & Ef. látice (\%) & 105,6 & & & & \\
\hline & P. de Minas & Trat. não aj. & $2.038 .858,8^{\text {*** }}$ & 10,3 & 3.052 & 95,1 & 2,55 \\
\hline & & Erro DBC & $99.782,9$ & & & & \\
\hline & & Ef. látice (\%) & - & & & & \\
\hline
\end{tabular}


Tabela 2. Continuação.

\begin{tabular}{|c|c|c|c|c|c|c|c|}
\hline Caráter & Local & Fonte de variação & Quadrado médio & $\mathrm{CV}_{\mathrm{e}}$ & Média & $\mathrm{h}^{2}$ & $\mathrm{~b}$ \\
\hline \multirow{7}{*}{$\begin{array}{l}\text { Altura } \\
\text { de plantas }\end{array}$} & & & \multicolumn{5}{|c|}{ Terceira época de semeadura } \\
\hline & \multirow[t]{3}{*}{ Lavras } & Trat. ajustado & $247,3 * *$ & \multirow[t]{3}{*}{9,2} & \multirow[t]{3}{*}{101,6} & \multirow[t]{3}{*}{64,4} & \multirow[t]{3}{*}{0,78} \\
\hline & & Erro efetivo & 87,9 & & & & \\
\hline & & Ef. látice (\%) & 130,6 & & & & \\
\hline & \multirow[t]{3}{*}{ P. de Minas } & Trat. não aj. & $259,1 * *$ & \multirow[t]{3}{*}{7,2} & \multirow[t]{3}{*}{110,9} & \multirow[t]{3}{*}{75,7} & \multirow[t]{3}{*}{1,02} \\
\hline & & Erro DBC & 62,9 & & & & \\
\hline & & Ef. látice (\%) & - & & & & \\
\hline \multirow{6}{*}{ Florescimento } & \multirow{3}{*}{ Lavras } & Trat. ajustado & $203,3^{* *}$ & \multirow{3}{*}{2,3} & \multirow{3}{*}{99,8} & \multirow{3}{*}{97,3} & \multirow[t]{3}{*}{3,5} \\
\hline & & Erro efetivo & 5,5 & & & & \\
\hline & & Ef. látice (\%) & 102,1 & & & & \\
\hline & \multirow[t]{3}{*}{ P. de Minas } & Trat. não aj. & $335,6 * *$ & \multirow[t]{3}{*}{6,1} & \multirow[t]{3}{*}{88,1} & \multirow[t]{3}{*}{91,5} & \multirow[t]{3}{*}{1,9} \\
\hline & & Erro DBC & 28,4 & & & & \\
\hline & & Ef. látice (\%) & - & & & & \\
\hline
\end{tabular}

${ }^{(1)}$ Erro do delineamento de blocos casualizados. ${ }^{*} \mathrm{e}^{* *}$ Significativo a $5 \%$ e $1 \%$ de probabilidade, respectivamente, pelo teste $\mathrm{F}$.

gunda época, apresentaram menores valores de $b$ (Tabela 2).

A avaliação das populações em épocas distintas de semeadura e em mais de um local permite ao melhorista conhecer melhor a reação dos genótipos frente às variações ambientais. Os resultados das interações encontrados neste trabalho, para os caracteres produtividade de grãos, altura de plantas e florescimento encontram-se na Tabela 3. A ausência de interação entre populações e locais para os três caracteres possibilita inferir que o comportamento desses genótipos foi semelhante nos dois locais.

A interação significativa entre populações x época $(\mathrm{PxE})$ foi importante, pois mostrou que o comportamento das populações diferiu nas três épocas de semeadura. Entretanto, observa-se que, para produtividade de grãos, a variância da interação $\mathrm{PxE}$ representou apenas $6,16 \%$ da variância genética de populações $\left(\sigma_{\mathrm{P}}^{2}\right)$. Para visualizar melhor esse fato, foi feito um estudo da interação de PxE duas a duas para cada local. As correlações entre as médias ajustadas das populações de uma época para outra são mostradas na Tabela 4. Verifica-se que houve correlação positiva e significativa entre as populações em todas as épocas duas a duas nos dois locais, cujas magnitudes variaram de 0,54 a 0,90 . A participação da parte complexa na interação $\mathrm{PxE}$ foi de maior magnitude em Lavras na $E_{1} v_{s} E_{2}(50,3 \%)$ e $E_{2}$ vs $E_{3}$ $(67,8 \%)$. Tais resultados foram coerentes com as estimativas de correlação, que foram mais baixas para essas duas situações. Assim, pode-se inferir que os efeitos da interação PxE foram mais pronunciados em Lavras, provavelmente em decorrência de características como fertilidade e preparo de solo, temperatura e distribuição das chuvas.

Em Patos de Minas, houve maior predominância da parte simples na interação $\mathrm{PxE}$, constatado tanto pelos resultados da correlação quanto pelas estimativas da variância da interação PxE $\left(\sigma^{2} \mathrm{PxE}\right)$, que representou pouco da variância genética de populações $\left(\sigma^{2}\right)$. A predominância da parte simples na interação facilita o trabalho do melhorista, pois permite selecionar materiais que tenham uma boa performance nos vários ambientes avaliados, ao passo que a presença da parte complexa indica que deve-se selecionar materiais específicos para cada ambiente.

A interação locais x épocas mostrada na Tabela 3 foi significativa para os três caracteres avaliados, indicando que as diferentes épocas de semeadura foram afetadas pelas características dos locais. Para produtividade de grãos, a estimativa da variância da interação $\operatorname{LxE}\left(\sigma_{\mathrm{LxE}}^{2}\right)$ foi bastante pronunciada, representando $39,47 \%$ da variância genética de populações $\left(\sigma_{\mathrm{P}}^{2}\right)$. O efeito dessa interação pode ser observado pelas médias das populações apresentadas na Tabela 2. Observa-se que à medida que se atrasou a época de semeadura em Lavras, a média das populações diminuiu, passando de $3.171 \mathrm{~kg} / \mathrm{ha}$ para $2.294 \mathrm{~kg} / \mathrm{ha}$. Em Patos de Minas aconteceu o contrário, a média foi maior $(3.052 \mathrm{~kg} / \mathrm{ha})$ quando a semeadura foi realizada mais tarde $\left(\mathrm{E}_{3}\right)$. Esses resulta- 
Tabela 3. Análise de variância conjunta para os caracteres produtividade de grãos ( $\mathrm{kg} / \mathrm{ha})$, altura de plantas $(\mathrm{cm})$ e florescimento (dia) avaliados em três épocas de plantio em Lavras e Patos de Minas, MG. 1996/97.

\begin{tabular}{|c|c|c|c|c|}
\hline \multirow{2}{*}{$\begin{array}{l}\text { Fonte de } \\
\text { variação }^{(1)}\end{array}$} & \multirow[t]{2}{*}{ GL } & \multicolumn{3}{|c|}{ QM } \\
\hline & & Produtividade de grãos & Altura de plantas & Florescimento \\
\hline Bloco/época/local & 12 & $3.976 .160,0 * *$ & $786,06^{* *}$ & $30,54 * *$ \\
\hline Local (L) & 1 & $128.838,0^{\mathrm{ns}}$ & $329,55^{\mathrm{ns}}$ & $3.383,67^{\mathrm{ns}}$ \\
\hline Época (E) & 2 & $1.693 .699,5^{\mathrm{ns}}$ & $1.136,57^{\mathrm{ns}}$ & $128,12^{\mathrm{ns}}$ \\
\hline$L \times E$ & 2 & $22.233 .508,8^{*}$ & $5.874,99 * *$ & $1.690,28 * *$ \\
\hline População (P) & 24 & $5.793 .461,4 * *$ & $847,81 * *$ & $1.256,42 * *$ \\
\hline $\mathrm{P} \times \mathrm{L}$ & 24 & $635.091,9^{\mathrm{ns}}$ & $52,69^{\mathrm{ns}}$ & $28,12^{\mathrm{ns}}$ \\
\hline$P \times E$ & 48 & $340.395,0^{*}$ & $122,95 * *$ & $25,94 * *$ \\
\hline$P \times E \times L$ & 48 & $547.144,6^{* *}$ & $75,99^{\text {ns }}$ & $29,23 * *$ \\
\hline Erro efetivo & 216 & $228.337,2$ & 56,10 & 13,13 \\
\hline Total & 449 & & & \\
\hline Média & & $2.780,73$ & 103,35 & 93,78 \\
\hline$\hat{\sigma}_{\mathrm{P}}^{2}$ & & $302.948,13$ & 40,270 & 68,36 \\
\hline$\hat{\sigma}_{\mathrm{PxE}}^{2}$ & & $\begin{array}{l}18.676,30 \\
\quad(6,16 \%)^{(2)}\end{array}$ & $\begin{array}{l}11,142 \\
(27,67 \%)\end{array}$ & $\begin{array}{l}2,14 \\
(3,13 \%)\end{array}$ \\
\hline$\hat{\sigma}_{\mathrm{PxL}}^{2}$ & & $\begin{array}{c}4.885,96 \\
(1,61 \%)\end{array}$ & 0 & 0 \\
\hline$\hat{\sigma}_{\mathrm{LxE}}^{2}$ & & $\begin{array}{r}119.590,28 \\
(39,47 \%)\end{array}$ & 0 & $\begin{array}{c}4,92 \\
(7,2 \%)\end{array}$ \\
\hline$\hat{\sigma}_{\mathrm{PLE}}^{2}$ & & $\begin{array}{c}53.134,56 \\
(17,5 \%)\end{array}$ & $\begin{array}{c}3,316 \\
(8,23 \%)\end{array}$ & $\begin{array}{l}2,68 \\
(2,69 \%)\end{array}$ \\
\hline $\mathrm{CV}_{\mathrm{E}}$ & & 17,18 & 7,24 & 3,86 \\
\hline $\mathrm{CV}_{\mathrm{g}}$ & & 20,00 & 6,14 & 8,81 \\
\hline $\mathrm{B}^{8}$ & & 1,16 & 0,85 & 2,28 \\
\hline $\mathrm{h}^{2}$ & & 94,12 & 85,50 & 97,93 \\
\hline
\end{tabular}

${ }^{(1)} \hat{\sigma}_{\mathrm{P}}^{2}$ : variância genética de populações; $\hat{\sigma}_{\mathrm{PxE}}^{2}$ : variância da interaç̃o entre populações e épocas; $\hat{\sigma}_{\mathrm{PxL}}^{2}$ : variância da interação entre populações e locais; $\hat{\sigma}_{\mathrm{LxE}}^{2}$ : variância da interação entre locais e épocas; $\hat{\sigma}_{\mathrm{PLE}}^{2}$ : variância da interação ente populações, locais e épocas. (2)Porcentagem em relação a $\hat{\sigma}_{\mathrm{P}}^{2}$, ${ }^{\text {ns }}$ ão-significativo. ${ }^{*} \mathrm{e}^{* *}$ Significativo a $5 \%$ e $1 \%$ de probabilidade, respectivamente, pelo teste $\mathrm{F}$.

dos ressaltam a discrepância das condições ambientais existentes nos dois locais.

O estudo dos parâmetros genéticos e fenotípicos contribui para melhorar a eficiência da avaliação do potencial genético das populações segregantes (Tabela 3). Nota-se que os resultados são satisfatórios para $\mathrm{CV}_{\mathrm{g}}$, as estimativas de $\mathrm{h}^{2}$ foram altas e os valores de $\mathrm{b}$ superiores a 1 , exceto para altura de plantas, o que mostra uma situação muito favorável para seleção. Uma maneira de aumentar o êxito da seleção simultânea de caracteres seria a utilização do índice de seleção. Na cultura do arroz, vários autores obtiveram resultados significativos utilizando índice de seleção (Morais, 1992; Rodrigues, 1995; Morais et al., 1997; Rangel et al., 1998).
Para a predição do potencial produtivo das populações segregantes, foi feita uma avaliação da produtividade por planta dentro de cada população aplicando o método de Jinks \& Pooni (1976), que objetiva encontrar linhagens que superem um valor padrão ou uma cultivar padrão. Para isso, considerouse como padrão $\overline{\mathrm{L}}=18,0 \mathrm{~g} /$ planta, pois a média da cultivar padrão Guarani $(15,42 \mathrm{~g} /$ planta) neste trabalho foi inferior à maior média encontrada entre as populações (17,02 g/planta). O mesmo método foi aplicado com soja (Triller, 1994) e feijão (Otubo et al., 1996; Abreu, 1997), mas não existem referências sobre a sua utilização com arroz.

Para a aplicação desse método, considera-se que a média da $\mathrm{F}_{\mathrm{ni}}$ representa a média das linhagens na 
Tabela 4. Estimativas da variância genética entre populações $\left(\sigma_{\mathrm{P}}^{2}\right)$, variância da interação população x época de semeadura $\left(\sigma_{\text {PxE }}^{2}\right)$ e estimativa da correlação (r) entre as médias ajustadas das populações de uma época para outra e contribuição da parte complexa na interação (C\%) para o caráter produtividade de grãos, em Lavras e Patos de Minas, MG. 1996/97.

\begin{tabular}{|c|c|c|c|c|c|}
\hline \multirow[t]{2}{*}{ Época $^{(1)}$} & \multirow[t]{2}{*}{ Variável } & \multicolumn{2}{|c|}{ Lavras } & \multicolumn{2}{|c|}{ Patos de Minas } \\
\hline & & $E_{2}$ & $E_{3}$ & $\mathrm{E}_{2}$ & $E_{3}$ \\
\hline \multirow[t]{2}{*}{$\mathrm{E}_{1}$} & $\sigma_{\mathrm{P}}^{2}$ & $354.450,38$ & $332.456,84$ & $270.594,00$ & $317.898,26$ \\
\hline & $\sigma_{\mathrm{PxE}}^{2}$ & $15.289,29$ & $30.028,73$ & $56.320,37$ & $72.442,45$ \\
\hline \multirow[t]{2}{*}{$\mathrm{E}_{2}$} & $\sigma_{P}^{2}$ & & $216.596,85$ & & $570.443,39$ \\
\hline & $\sigma_{\mathrm{PxE}}^{2}$ & & $28.901,18$ & & $112.986,16$ \\
\hline \multirow[t]{2}{*}{$\mathrm{E}_{1}$} & $\mathrm{r}$ & $0,66^{* *} *$ & $0,71 * *$ & $0,72 * *$ & $0,71 * *$ \\
\hline & $\mathrm{C} \%$ & 50,3 & 41,4 & 28,6 & 24,5 \\
\hline \multirow{2}{*}{$\mathrm{E}_{2}$} & $\mathrm{r}$ & & $0,54 * *$ & & $0,90 * *$ \\
\hline & $\mathrm{C} \%$ & & 67,8 & & 30,0 \\
\hline
\end{tabular}

${ }^{(1)} \mathrm{E}_{1}, \mathrm{E}_{2}, \mathrm{E}_{3}$ : primeira, segunda e terceira época de semeadura, respectivamente. ${ }^{*}$ Significativo a $1 \%$ de probabilidade

$\mathrm{F}_{\infty}$ (Jinks \& Pooni, 1976). Para que isso seja válido, é necessário que haja a predominância da ação gênica aditiva. Em arroz, observa-se uma predominância desse tipo de ação gênica para caracteres quantitativos (Lopes, 1984), sendo a ação não-aditiva observada em alguns casos, mas em menores magnitudes (Ahmad et al., 1986; Morais, 1992). Neste trabalho não foram considerados os efeitos de dominância em nenhuma das gerações avaliadas.

Na Tabela 5, encontram-se os resultados da média geral das populações, média por planta, variância genética, fenotípica e ambiental, herdabilidade de indivíduo e os valores de $\mathrm{Z}$ com suas respectivas porcentagens, que indicam a probabilidade de extrair linhagens superiores em uma determinada população. Observa-se que as populações que mais se destacaram em produtividade de grãos foram CNAx 5901, CNAx 5496, CNAx 6106 e CNAx 6001, com médias variando de 3.593 a $3.334 \mathrm{~kg} / \mathrm{ha}$. As populações que apresentaram o pior desempenho médio foram CNAx 6063 e CNAx 6102, cujas médias foram de 1.859 e $1.269 \mathrm{~kg} / \mathrm{ha}$, respectivamente. Observa-se que a média da cultivar Guarani $(3.855 \mathrm{~kg} / \mathrm{ha}$ ) foi superior à média de todas a populações avaliadas. No Estado de Minas Gerais, a Guarani foi uma das cultivares mais plantadas, pelo seu elevado potencial produtivo e comportamento estável (Tabela 6), embora os grãos não sejam classificados como agulhinha.
Merece ser destacado o comportamento médio das populações em relação ao número de genitores envolvidos no cruzamento e a geração em que as populações encontravam-se no presente trabalho. Pôdese notar que esses dois fatores não foram determinantes no desempenho final das populações, ou seja, as três melhores populações representavam tanto cruzamentos múltiplos como biparental e também as três gerações $\left(\mathrm{F}_{3}, \mathrm{~F}_{4}\right.$ e $\left.\mathrm{F}_{5}\right)$ estudadas (Tabela 1). Esse resultado reforça o fato de que um cruzamento biparental pode ser tão promissor quanto um cruzamento múltiplo, desde que os genitores sejam bem escolhidos e a população segregante bem conduzida. Os cruzamentos biparentais que mais se destacaram neste trabalho foram CNAx 4442-6-1-B-1 x 4001.BC 92.93 e CNA 8200 x IAC 1203, sendo identificados como populações CNAx 6001 e CNAx 6106, respectivamente.

Em relação às médias de produção de grãos por planta, verifica-se que houve uma variação significativa entre as populações CNAx 6102 (8,18 g/planta) e CNAx 5496 (17,02 g/planta). A correlação encontrada entre a média geral da população e a média por planta de cada população foi de $0,77 * *$, indicando que, de maneira geral, as plantas amostradas aleatoriamente dentro das populações refletiram a média geral da população. Já a variância genética de cada população correlacionou-se negativamente com a média geral das populações $(-0,62 * *)$, ou seja, médias altas coincidiram com variâncias genéticas 
baixas, o que dificulta a seleção que busca conciliar altas variâncias genéticas com altas médias. Normalmente, pode haver a ocorrência de erros associados às estimativas da variância genética (Vello \& Vencovsky, 1974), principalmente para variância no âmbito de indivíduo.

Os resultados encontrados neste trabalho em relação à probabilidade de extrair linhagens superiores de uma determinada população indicam como mais promissora a população CNAx 5496 (42,47\%), seguida da CNAx 6001 (20,33\%), ambas também entre as mais produtivas citadas anteriormente. Por sua vez, as populações menos promissoras foram CNAx 5995 (8,08\%), CNAx 6065 (6,94\%), CNAx 6063 (8,08\%) e CNAx 6102 (4,27\%) (Tabela 5). Tais observações podem ser corroboradas pe- las correlações negativas e significativas encontradas entre a média geral da população e o valor de $Z$ $\left(\mathrm{r}=-0,53^{* *}\right)$, e entre a média por planta da população e o valor de $Z\left(r=-0,91^{* *}\right)$. É importante esclarecer que valores menores de $Z$ correspondem a valores altos de probabilidade. Esses resultados confirmam o que vários autores citam, ou seja, a média é um bom referencial para selecionar os materiais superiores e também uma medida fácil de se obter, principalmente para a cultura do arroz, cuja análise individual é dificultada pelo intenso perfilhamento.

Considerando a média e a variância das populações, deve-se dar prioridade à média, uma vez que algumas populações apresentavam altas variâncias mas médias muito baixas. A maior eficiência da média foi a correlação negativa e significativa com o

Tabela 5. Média da população, média por planta $\left(\overline{\mathrm{F}}_{\mathrm{n}}\right)$, variâncias genética $\left(\sigma_{\mathrm{G}}^{2}\right)$, ambiental $\left(\sigma_{\mathrm{E}}^{2}\right)$, fenotípica $\left(\sigma^{2} \mathrm{Fn}\right)$, herdabilidade $\left(\mathrm{h}^{2}\right)$, valor de $\mathrm{Z}$ para $\overline{\mathrm{L}}=18,0 \mathrm{~g}$ /planta e suas respectivas probabilidades ( $\mathrm{P} \%$ ), para o caráter produtividade de grãos. Lavras e Patos de Minas, MG. 1996/97.

\begin{tabular}{|c|c|c|c|c|c|c|c|c|}
\hline População (geração) & $\begin{array}{l}\text { Média } \\
\text { (kg/ha) }\end{array}$ & $\begin{array}{c}\overline{\mathrm{F}}_{\mathrm{n}} \\
\text { (g/planta) }\end{array}$ & $\sigma_{\mathrm{G}}^{2}$ & $\sigma_{\mathrm{E}}^{2}$ & $\sigma_{\mathrm{Fn}}^{2}$ & $h^{2}$ & $\begin{array}{c}\mathrm{Z} \\
(\overline{\mathrm{L}}=18,0 \mathrm{~g} / \\
\text { planta })\end{array}$ & $\mathrm{P}(\%)$ \\
\hline Guarani & 3.855 & 15,42 & - & 25,51 & 25,21 & - & - & - \\
\hline CNAx $5901\left(\mathrm{~F}_{4}\right)$ & 3.593 & 13,84 & 1,32 & 22,13 & 20,81 & 6,34 & 0,92 & 17,88 \\
\hline CNAx $5496\left(\mathrm{~F}_{5}\right)$ & 3.551 & 17,02 & 4,20 & 22,21 & 26,42 & 15,90 & 0,19 & 42,47 \\
\hline CNAx $6106\left(\mathrm{~F}_{3}\right)$ & 3.440 & 13,66 & 1,65 & 20,94 & 19,29 & 8,55 & 0,99 & 15,11 \\
\hline CNAx $6001\left(\mathrm{~F}_{3}\right)$ & 3.334 & 13,09 & 6,57 & 22,10 & 28,67 & 22,92 & 0,87 & 20,33 \\
\hline CNAx $5939\left(\mathrm{~F}_{4}\right)$ & 3.085 & 12,71 & 5,90 & 19,27 & 25,17 & 23,44 & 1,04 & 14,92 \\
\hline Canastra & 3.045 & 13,36 & - & 27,22 & 27,22 & - & - & - \\
\hline CNAx $5983\left(\mathrm{~F}_{4}\right)$ & 3.001 & 14,53 & 1,29 & 25,12 & 26,41 & 4,88 & 0,67 & 25,14 \\
\hline CNAx $6062\left(\mathrm{~F}_{3}\right)$ & 2.929 & 13,43 & 4,69 & 22,59 & 27,28 & 17,19 & 0,84 & 20,05 \\
\hline CNAx $5482\left(\mathrm{~F}_{5}\right)$ & 2.921 & 13,61 & 12,09 & 22,19 & 34,28 & 35,27 & 0,74 & 22,96 \\
\hline CNAx $5500\left(\mathrm{~F}_{5}\right)$ & 2.910 & 13,43 & 3,88 & 22,96 & 26,84 & 14,46 & 0,88 & 18,94 \\
\hline CNAx $6090\left(\mathrm{~F}_{3}\right)$ & 2.844 & 12,82 & 7,12 & 19,41 & 26,53 & 26,84 & 0,95 & 17,11 \\
\hline CNAx $5945\left(\mathrm{~F}_{4}\right)$ & 2.775 & 13,95 & 7,25 & 23,91 & 31,16 & 23,27 & 0,71 & 23,89 \\
\hline CNAx $5493\left(\mathrm{~F}_{5}\right)$ & 2.724 & 14,85 & 18,83 & 28,93 & 47,75 & 39,43 & 0,45 & 32,64 \\
\hline CNAx $5987\left(\mathrm{~F}_{4}\right)$ & 2.722 & 13,54 & 11,81 & 20,44 & 32,25 & 36,62 & 0,77 & 22,06 \\
\hline CNAx $5883\left(\mathrm{~F}_{4}\right)$ & 2.709 & 12,78 & 9,68 & 18,71 & 28,39 & 34,10 & 0,96 & 16,85 \\
\hline CNAx $5494\left(\mathrm{~F}_{5}\right)$ & 2.652 & 12,34 & 14,49 & 16,79 & 31,27 & 46,34 & 1,00 & 15,86 \\
\hline CNAx $6067\left(\mathrm{~F}_{3}\right)$ & 2.603 & 12,26 & 1,39 & 19,93 & 21,32 & 6,52 & 1,21 & 11,31 \\
\hline CNAx $5996\left(\mathrm{~F}_{3}\right)$ & 2.591 & 14,15 & 9,40 & 26,95 & 36,35 & 25,86 & 0,61 & 27,09 \\
\hline CNAx $5995\left(\mathrm{~F}_{3}\right)$ & 2.425 & 10,79 & 7,97 & 15,46 & 23,43 & 34,02 & 1,40 & 8,08 \\
\hline CNAx $6190\left(\mathrm{~F}_{3}\right)$ & 2.346 & 13,93 & 14,64 & 21,80 & 36,44 & 40,18 & 0,63 & 26,43 \\
\hline CNAx $5949\left(\mathrm{~F}_{4}\right)$ & 2.221 & 12,73 & 17,80 & 19,35 & 37,14 & 47,93 & 0,84 & 20,04 \\
\hline CNAx $6065\left(\mathrm{~F}_{3}\right)$ & 2.115 & 10,27 & 10,72 & 12,82 & 23,54 & 45,54 & 1,48 & 6,94 \\
\hline CNAx $6063\left(\mathrm{~F}_{3}\right)$ & 1.859 & 10,82 & 6,70 & 16,84 & 23,54 & 28,46 & 1,40 & 8,08 \\
\hline CNAx $6102\left(\mathrm{~F}_{3}\right)$ & 1.269 & 8,18 & 18,42 & 7,78 & 26,20 & 70,31 & 1,72 & 4,27 \\
\hline
\end{tabular}


Tabela 6. Resultado da análise de estabilidade pelo método de Annicchiarico (1992) para as populações de arroz de sequeiro avaliadas em Lavras e Patos de Minas em três épocas distintas de semeadura. 1996/97.

\begin{tabular}{|c|c|c|c|c|}
\hline Populações & Média & $\mathrm{I}(\mathrm{i})^{(1)}$ & Desvio padrão & Média $(\%)$ \\
\hline CNAx 5482 & $2.921,33$ & 85,00 & 20,60 & 106,34 \\
\hline CNAx 5493 & $2.723,98$ & 91,83 & 5,97 & 98,02 \\
\hline CNAx 5494 & $2.652,17$ & 85,31 & 10,15 & 95,83 \\
\hline CNAx 5496 & $3.550,77$ & 114,80 & 11,90 & 127,13 \\
\hline CNAx 5500 & $2.909,75$ & 93,05 & 10,88 & 104,33 \\
\hline CNAx 5883 & $2.708,60$ & 80,19 & 17,24 & 98,06 \\
\hline CNAx 5901 & $3.593,05$ & 115,12 & 13,15 & 128,75 \\
\hline CNAx 5939 & $3.084,85$ & 98,72 & 11,09 & 110,21 \\
\hline CNAx 5945 & $2.775,45$ & 88,67 & 11,15 & 100,23 \\
\hline CNAx 5949 & $2.221,48$ & 69,37 & 10,58 & 80,34 \\
\hline CNAx 5983 & $3.001,12$ & 97,69 & 9,44 & 107,48 \\
\hline CNAx 5987 & $2.722,38$ & 80,93 & 17,75 & 99,33 \\
\hline CNAx 6190 & $2.346,48$ & 75,27 & 9,55 & 85,16 \\
\hline CNAx 5995 & $2.424,60$ & 66,61 & 19,10 & 86,41 \\
\hline CNAx 5996 & $2.590,93$ & 82,35 & 10,14 & 92,86 \\
\hline CNAx 6067 & $2.602,52$ & 85,33 & 7,85 & 93,46 \\
\hline CNAx 6090 & $2.843,57$ & 90,45 & 11,42 & 102,28 \\
\hline CNAx 6102 & $1.268,83$ & 39,93 & 5,91 & 46,05 \\
\hline CNAx 6106 & $3.439,63$ & 111,43 & 11,54 & 123,39 \\
\hline CNAx 6001 & $3.333,82$ & 103,18 & 14,95 & 118,67 \\
\hline CNAx 6062 & $2.929,32$ & 101,09 & 4,41 & 105,67 \\
\hline CNAx 6063 & $1.858,78$ & 57,75 & 8,74 & 66,82 \\
\hline CNAx 6065 & $2.114,55$ & 62,52 & 13,40 & 76,42 \\
\hline Guarani & $3.855,10$ & 110,98 & 25,25 & 137,14 \\
\hline Canastra & $3.045,38$ & 89,41 & 19,51 & 109,63 \\
\hline
\end{tabular}

(1)Índice de confiança; significativo a $15 \%$.

valor de Z, pois quanto menor o valor de Z maior é a probabilidade de selecionar as linhagens superiores na $\mathrm{F}_{\infty}$. A variância genética no âmbito de indivíduo é uma estimativa passível de erros e neste trabalho não se mostrou um bom preditor do potencial genético das populações. Logo, $\mathrm{h}^{2}$ não poderia ser recomendada neste trabalho, uma vez que correlacionou-se negativamente com as médias e não apresentou correlação com $Z$.

Para confirmação da eficiência desse método de seleção, foram realizados estudos de estabilidade com as populações. Para tanto, foram selecionadas duas populações que apresentavam o melhor desempenho médio (CNAx 5496 e CNAx 6001) e duas com o pior desempenho (CNAx 6102 e CNAx 6063). O método utilizado foi o de Annicchiarico (1992). Estimase um índice de confiança (I(i)) que considera o risco de uma determinada população apresentar desem- penho abaixo de um dado padrão, como, por exemplo, a média dos materiais avaliados. Os valores obtidos são apresentados na Tabela 6. Observando os valores de I(i), pode-se relatar que somente as populações CNAx 5496, CNAx 5901, CNAx 6106, CNAx 6001, CNAx 6062 e a testemunha Guarani possuem $85 \%$ de probabilidade de apresentarem produtividade $14,8,15,1,11,4,3,2,1,1$ e $11 \%$, respectivamente, superior à média do ambiente, sendo portanto constituídas de materiais mais estáveis frente às oscilações ambientais. Nota-se que as duas populações (CNAx 5496 e CNAx 6001) consideradas melhores estão incluídas entre os materiais mais estáveis. As populações escolhidas como as piores (CNAx 6102, CNAx 6063) foram as menos estáveis, pois apresentaram $85 \%$ de probabilidade de apresentarem produtividade em torno de $60 \%$ e $42 \%$, inferior à média do ambiente. 


\section{Conclusões}

1. O método de Jinks \& Pooni é uma alternativa eficiente na escolha de populações mais promissoras em arroz.

2. A presença da interação populações segregantes $\mathrm{x}$ época de semeadura e locais $\mathrm{x}$ épocas de semeadura mostra a necessidade de avaliar as populações em mais de um ambiente.

\section{Referências}

ABREU, A. de F. B. Predição do potencial genético de populações segregantes do feijoeiro utilizando genitores inter-raciais. Lavras : UFLA, 1997. 80 p. Tese de Doutorado.

AHMAD, L.; ZAKRI, A. H.; JALANI, B. S.; OMAR, D Detection of additive and non-additive variation in rice In: INTERNATIONAL RICE RESEARCH INSTITUTE (Los Baños, Filipinas). Rice genetics: proceedings of the International Rice Genetics Symposium. Los Baños, 1986. p. $555-564$.

ANNICCHIARICO, P. Cultivar adaptation and recommendation from alfalfa trials in Northern Italy. Journal of Genetic \& Breeding, Rome, v. 46, n. 3, p. 269278, Sept. 1992

CRUZ, C. D.; CASTOLDI, F. L. Decomposição da interação genótipo $\mathrm{x}$ ambiente em partes simples e complexa. Revista Ceres, Viçosa, v. 38, n. 219, p. 422-430, 1991

JINKS, J. L.; POONI, H. S. Predicting the properties of recombinant inbreed lines derived by single seed descent. Heredity, Oxford, v. 36, n. 2, p. 243-266, 1976

LOPES, A. M. Análise genética dos componentes de produção num dialelo entre seis cultivares de arroz (Oryza sativa L.) em dois regimes hídricos. Viçosa : UFV-Imprensa Universitária, 1984. 135 p. Tese de Doutorado.

MELO, L. C.; SANTOS, J. B.; RAMALHO, M. A. P. Choice of parents to obtain common bean (Phaseolus vulgaris) cultivars tolerant to low temperatures at the adult stage. Brazilian Journal of Genetics, Ribeirão Preto, v. 20, n. 2, p. 283-292, 1997.

MORAIS, O. P. Análise multivariada da divergência genética dos progenitores, índices de seleção e seleção combinada numa população de arroz oriunda de intercruzamentos, usando macho-esterilidade. Viçosa UFV, 1992. 251 p. Tese de Doutorado.
MORAIS, O. P.; CASTRO, E. da M. de; SANT'ANA, E. P. Selección recurrente en arroz de secano en Brasil. In: CENTRO INTERNACIONALDEAGRICULTURA TROPICAL (Cali, Colômbia). Selección recurrente en arroz. Cali, 1997. 240 p.

MORAIS, O. P.; SILVA, J. C.; VIEIRA, C.; SOARES, P. C.; GALVÃO, J. D. Adaptabilidade e estabilidade de comportamento de variedades e linhagens de arroz irrigado em Minas Gerais. Revista Ceres, Viçosa, v. 28, n. 156, p. $150-158,1981$

MORAIS, O. P.; SOARES, P. C.; SILVA, J. C.; VIEIRA, C. Adaptabilidade e estabilidade de comportamento de onze variedades de arroz de sequeiro no Triângulo Mineiro e Alto Paranaíba. Pesquisa Agropecuária Brasileira, Brasília, v. 17, n. 5, p. 721-728, maio 1982.

OTUBO, S. T. Controle genético da tolerância do feijoeiro a baixas temperaturas na fase de germinação Lavras : UFLA, 1994. 51 p. Dissertação de Mestrado.

OTUBO, S. T.; RAMALHO, M. A. P.; ABREU, A. de F. B.; SANTOS, J. B. dos. Genetic control of low temperature tolerance in germination of the common bean (Phaseolus vulgaris L.). Euphytica, Dordrecht, v. 89, p. $313-317,1996$

RAMALHO, M. A. P.; SANTOS, J. B.; ZIMMERMANN, M. J. Genética quantitativa em plantas autógamas: aplicações ao melhoramento do feijoeiro. Goiânia : Ed. da UFG, 1993. 271 p.

RANGEL, P. H. N.; ZIMMERMANN, F. J. P.; NEVES, P. C. F. Estimativas de parâmetros genéticos e resposta à seleção nas populações de arroz irrigado CNA-IRAT 4PR e CNA-IRAT 4ME. Pesquisa Agropecuária Brasileira, Brasília, v. 33, n. 6, p. 905-912, jun. 1998.

RODRIGUES, R. E. S. Estimação de parâmetros genéticos e de respostas à seleção na população de arroz irrigado CNA 1. Goiânia : UFG, 1995. 69 p. Dissertação de Mestrado.

SANTOS, P. G. Estimativas de parâmetros genéticos e fenotípicos em populações segregantes de arroz irrigado por inundação. Lavras : UFLA, 1996. 72 p. Dissertação de Mestrado.

SNAPE, J. W. Predicting the frequencies of transgressive segregants for yield and yield components in wheat. Theoretical and Applied Genetics, Berlin, v. 62, n. 2, p. 127-134, 1982 
TOLEDO, J. F. F. de. Biometrical studies of inbreed lines and their hybrids. Birmingham : University of Birmingham, 1986. 139 p. Ph.D. Thesis

TOLEDO, J. F. F. de. Predicting the inbreeding and the outcrossing potential of soybean (Glycine max L.) varieties Revista Brasileira de Genética, Ribeirão Preto, v. 10, n. 3 , p. $543-558$, set. 1987

TOLEDO, J. F. F. de. Quantitative genetics in soybean breeding. In: CONFERENCIA MUNDIAL DE INVESTIGACIÓN IN SOJA, 4., 1989, Buenos Aires. Anais... Buenos Aires : Soybean Association of Argentina, 1989. p. 909-914.
TRILLER, C. Previsão do potencial genético de cruzamentos em soja pela geração $\mathbf{F}_{3}$. Londrina : UEL/ Embrapa/IAPAR, 1994. 133 p. Dissertação de Mestrado.

VELLO, N. A.; VENCOVSKY, R. Variâncias associadas às estimativas de variância genética e coeficiente de herdabilidade. In: ESCOLA SUPERIOR DE AGRICULTURA LUIZ DE QUEIROZ (Piracicaba, São Paulo). Relatório científico de 1974. Piracicaba, 1974. p. 238-248. (Relatório, 8).

VENCOVSKY, R.; BARRIGA, P. Genética biométrica no fitomelhoramento. Ribeirão Preto : Sociedade Brasileira de Genética, 1992. 496 p. 